\title{
Ewing's sarcoma of the mandible-A rare case report
}

\author{
Shahid Hassan, M.D.S., BH Sripathi Rao, M.D.S., Gunachandra Rai, M.D.S. \\ Department of Oral \& Maxillofacial Surgery, Yenepoya Dental College \& Hospital
}

\section{A B S T R A C T}

Ewing's sarcoma is a malignant tumor of bones that primarily affects children and young adults. The true origin of this small round cell lesion still remains controversial.It was originally described by James Ewing in 1921 as arising from undifferentiated osseous mesenchymal cells, however, recent studies suggests that Ewing's tumor might be neuroectodermally derived from various degrees of differentiation of the primitive neural tissues. This paper reports a rare case of ES of the mandible in a 11 -year-old girl, which had been previously misdiagnosed and treated as a dental abscess. In the clinical examination, a hard immobile expansive mass of $2 \mathrm{~cm}$ diameter was observed on the left side of the mandible. Radiographic examination revealed a diffuse radiolucent lesion with ill-defined borders and wide vestibular bone plate destruction. Microscopically, the tumor was composed by monotonous small round cells that exhibited immunoreactivity for CD99, vimentin and desmin. Surgical resection of mandible followed by mandibular reconstruction was adopted. The patient was subjected to multiagent chemotherapy with Vincristine [VC],Dactinomycin [AC],Cyclophosphamide [CP] and Doxorubicin [AD]). JMS $2011 ; 14(2): 68-70$

Key Word: Ewing's sarcoma, mandible, immunohistochemistry.

Ewing's sarcoma (ES) is a rare malignant small round cell tumor that primarily affects the skeletal system. It accounts for 4 to $10 \%$ of all types of bone cancer, with long bones and pelvis being the most common locations. It affects mainly adolescents and young adults and is rarely seen before the age of 5 and after age of 30. Clinically, this tumor has an aggressive behavior characterized by rapid growth and high probability of micrometastasis at diagnosis.

\section{Case Report}

An 11-year-old girl visited to Department of Oral \& Maxillofacial Surgery Yenepoya dental college and hospital Deralakatte, Mangalore, with the complaint of swelling on the left side of the face (Fig.1). The swelling started 6 months

Correspondence and Reprint request to:

Prof. Gunachandra Rai, MDS

Professor, Department of Oral \& Maxillofacial Surgery,

Yenepoya Dental College \& Hospital back which gradually enlarged to present size. On examination, extra orally a diffuse swelling was seen on left side of face which extended superiorly up to ala tragus line, Anteriorly from about $2 \mathrm{~cm}$ posterior to commisure of mouth to angle of the mandible. Intra oral examination revealed mixed dentition and swelling extending from left mandibular canine to left mandibular second molar (Fig.1).

On palpation the swelling was non-tender and hard in consistency. Bi-digital palpation revealed expansion of the buccal plate, no perforation of buccal/lingual cortex and no paresthesia of mucosa or lower lip were seen. Grade III mobility was seen on all the teeth from left mandibular canine to left mandibular second molar, which were non tender on percussion. The intra oral periapical radiograph shows radiolucency near the mandibular teeth (Fig 2). The CT Scan findings revealed: an ill-defined expansile cystic lesion were seen in the body of the mandible on Lt side and thinning \& erosion of buccal cortex was also noticed (Fig 2).

The results of hematological and biochemical investigations were within normal limits. Under local 


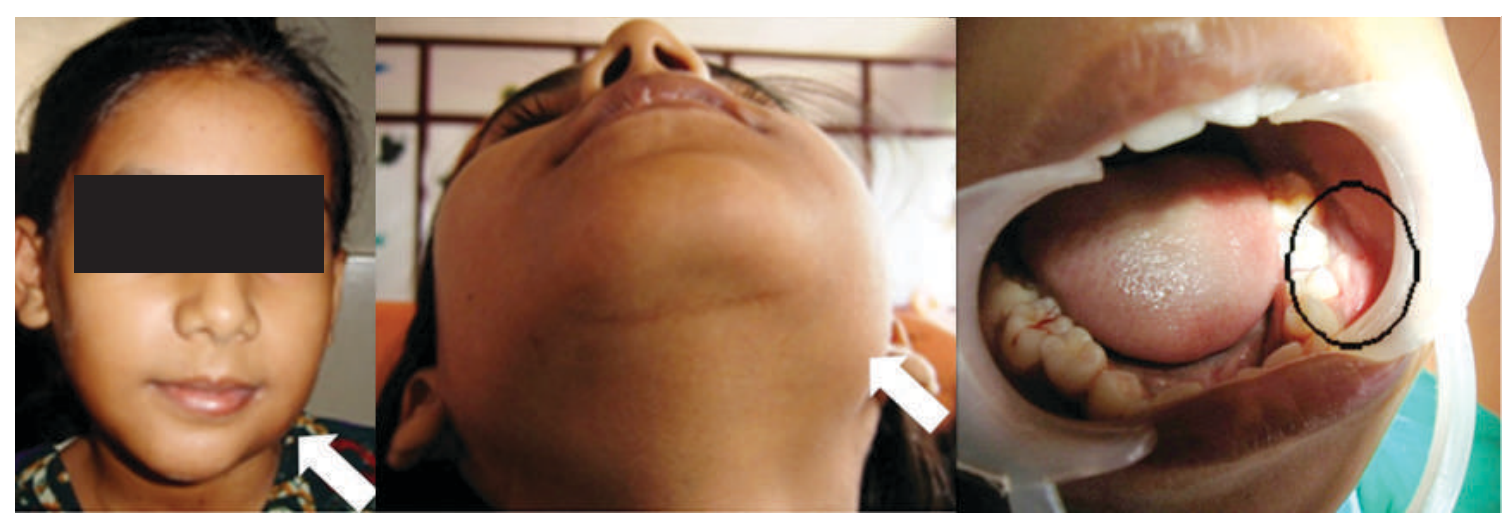

FIGURE 1: Showing the swelling: extra oral (white arrows) and intra-Oral (black swelling)

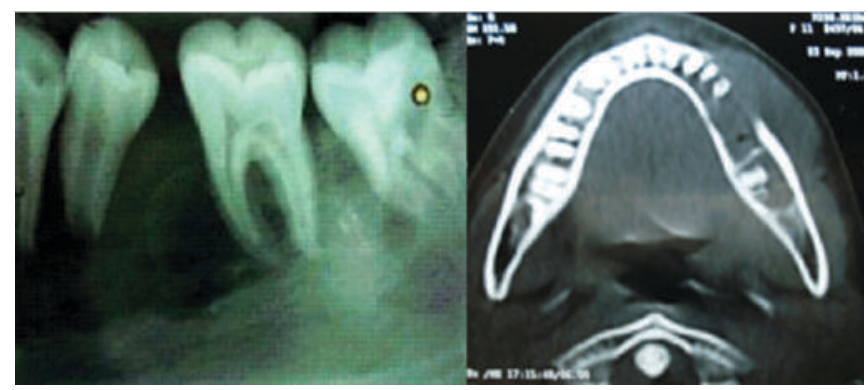

FIGURE 2: The intra oral periapical radiograph showing radiolucency near the mandibular teeth

anesthesia, extraction of the mobile teeth left mandibular first molar was performed and the tissue sample obtained through the extraction socket was sent for histopathological examination. (Fig. 3)

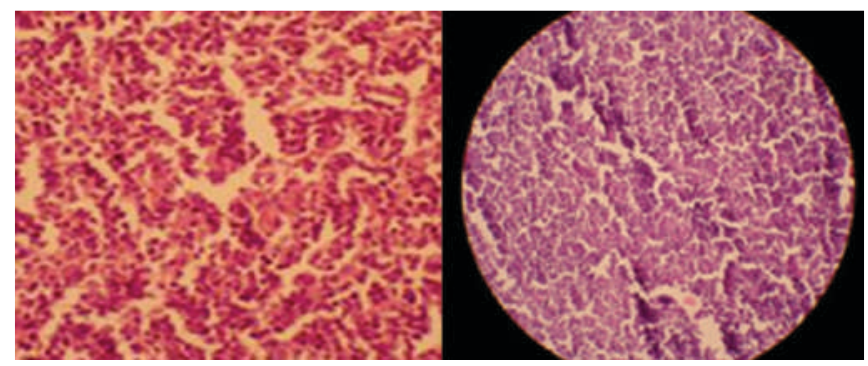

FIGURE 3: Microscopic picture under 10x (right panel) and 40x (Left panel) demonstrating the sheets of monotonous, round cells containing single, round to oval nucleus.

On microscopic examination, H\&E stained sections showed sheets of uniform, small, round cells arranged in diffuse pattern, with indistinct outline, scanty cytoplasm, and well-defined nuclear outline, with round to oval nucleus and inconspicuous nucleoli. Mitotic figures were rare. A diagnosis of malignant small round cell tumor was made based on the above histopathological findings. A panel of immunohistochemical markers namely CD99, CD3, CD20, CHR, MPO, desmin, and SYN were used to rule out other small round cell tumors. Tissue sections showed positive expression for CD99 (MIC2), with characteristic membranous pattern and an IMPRESSION drawn was Conformation of diagnosis of EWINGS SARCOMA. Bone Scan was also advised which revealed Reduced uptake over the body of left mandible without rim of enhanced uptake. No evidence of distal bone involvement. A surgical procedure was performed to resect the malignant tissue with generous surgical margins and to reconstruct the resulting defect. The patient underwent a segmental resection of left side including body of mandible. The bony defect was reconstructed using titanium reconstruction plate (Fig. 4). The postoperative panoramic radiograph revealed a wellaligned reconstruction with intact plating (Fig 5).

Adjuant Chemotherapy comprised 20 weeks (3 Cycles) of treatment with a four-drug regimen which were Vincristine [VC],Dactinomycin [AC],Cyclophosphamide $[\mathrm{CP}]$ and Doxorubicin $[\mathrm{AD}]$ after surgery.

\section{Discussion}

Ewing's sarcoma (ES) is a rare malignant small round cell tumor that primarily affects the skeletal system. It accounts for 4 to $10 \%$ of all types of bone cancer, with long bones and pelvis being the most common locations. It affects mainly adolescents and young adults and is rarely seen before the age of 5 and after age of 30. Clinically, this tumor has an aggressive behavior characterized by rapid growth and high probability of micrometastasis at diagnosis. ${ }^{1} \mathrm{ES}$ is a malignant neoplasm that primarily affects long bones of the extremities with nearly $50 \%$ of reported cases involving the femur and pelvis. $^{2}$ It exhibits a marked predilection for whites and is rarely seen among blacks. ${ }^{3,4}$ The majority of the patients affected are between the ages 5 and 20, whereas the disease is distinctly uncommon in individuals before age 5 and after age 30 (braz dent). ES arising from the bones of the head and neck region is exceedingly uncommon. When it occurs in the jaw, mandible is more frequently affected than the maxilla. ${ }^{1,2}$

Ewing's sarcoma is usually sensitive to chemotherapy and radiotherapy. Modern treatments are based on combined Modality of treatment Local therapy (surgery and/or 


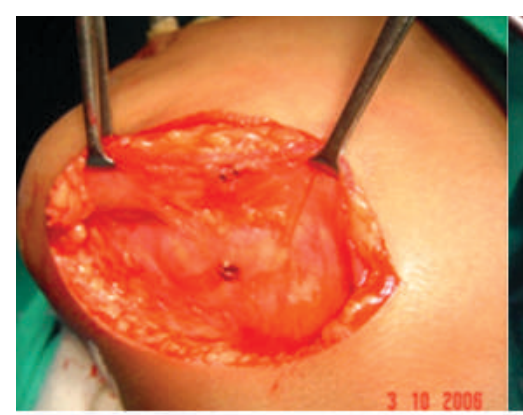

Incision

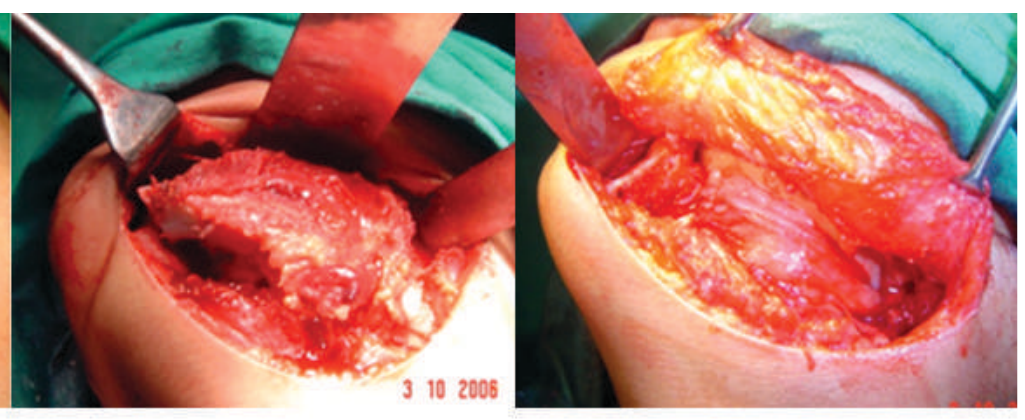

Exposure \& Resection

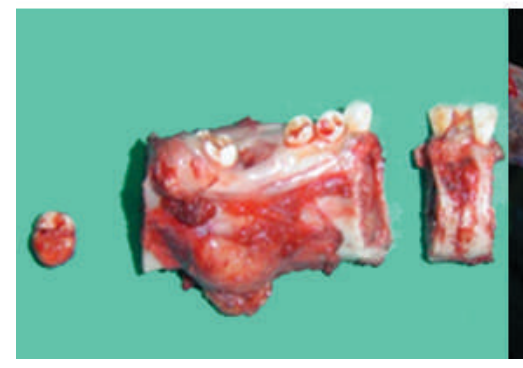

Excised specimen

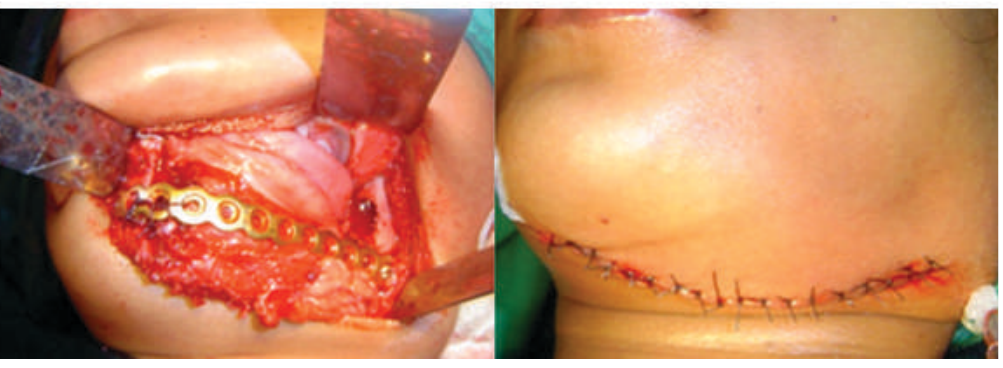

Reconstruction
Closure

FIGURE 4: Reconstruction surgery.

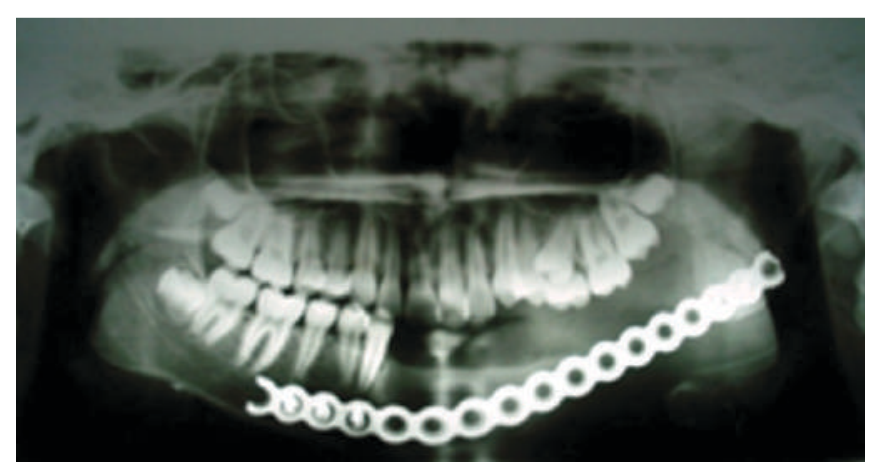

FIGURE 5: Six months post operative orthopantamograph

radiotherapy to the main tumour) followed by Chemotherapy (For management of micrometastasis). The present case was positive for CD99, vimentin and desmin and negative for other immunomarkers, leading to a diagnosis of ES. ${ }^{1}$

In conclusion, oral Ewing sarcoma is a rare tumour entity which can mimic odontogenic inflammation. If root amputation surgery is performed due to an extensive radiolucent lesion, histopathological evaluation should be mandatory. Primary bony reconstruction bears the risk of second intervention when dealing with ES in the mandible. ${ }^{5}$

\section{References}

1. Brazão-Silva MT, Fernandes AV, De Faria PR, Cardoso SV, Loyola AM. Ewing's Sarcoma of the Mandible in a Young Child. Braz Dent J 2010; 21(1):74-79.

2. Lopes SLPC, DeAlmida SM,Costa ALF,Zanardi VA,Cendes F.Imaging findings of Ewings Sarcoma in the Mandible. Journal of Oral Science 2007;49(2):167-71.

3. Talesh KT, Kalantar Motamedi MH, Jeihounian M. Ewing's Sarcoma of the Mandibular Condyle: Report of a Case. J Oral Maxillofac Surg 2003;61:1216-19.

4. Richard Berk, Allen Heller, Debra Heller, Steven Schwartz, Edward A. Klein. Ewing's sarcoma of the mandible. Oral Surg Oral Med Oral Pathol Oral Radiol Endod 1995;79:159-62.

5. Martin Gosau, Daniel Baumhoer, Stefan Ihrler, Johannes Kleinheinz, Oliver Driemel. Ewing sarcoma of the mandible mimicking an odontogenic abscess - a case report. Head E Face Medicine 2008;4:24. 